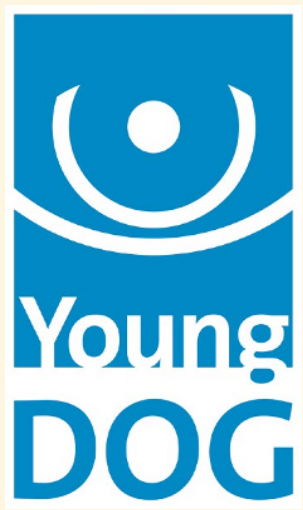

Die AG Young DOG richtet sich an junge Assistenzärzte und Nachwuchswissenschaftler. Sie fokussiert ihre Aktivitäten auf die Förderung des wissenschaftlichen Nachwuchses in der Ophthalmologie und die Vereinbarkeit von Klinik, Forschung und Familie.

Die Mitgliedschaft in der AG ist den Mitgliedern der DOG vorbehalten. Mitglied werden können Assistenzärzte, habilitierte Ärzte und Wissenschaftler sowie Professoren bis W 2.

Nähere Information zur AG Young DOG finden Sie unter http://www. dog.org/?cat=137.

\section{Corinna von Goscinski}

Zentrum für Augenheilkunde, Uniklinik Köln, Köln, Deutschland

\section{Korrespondenzadresse:}

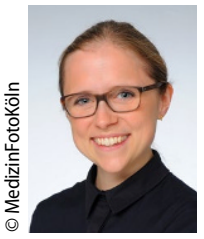

Corinna von Goscinski

Zentrum für Augenheilkunde,

Uniklinik Köln

Kerpener Str. 62 ,

50924 Köln, Deutschland

corinna.von-goscinski@uk-koeln.de

Ophthalmologe 2019 • 116:1090

https://doi.org/10.1007/s00347-019-

0929-8

Online publiziert: 4. Juli 2019

(c) Springer Medizin Verlag GmbH,

ein Teil von Springer Nature 2019

\title{
Praxisrotation während der Facharztausbildung an einer Universitätsklinik
}

„Wieso denn jetzt in die Praxis, du bist doch an der Uniklinik?" - Mit dieser Frage wurde ich nicht nur einmal konfrontiert, als ich beschlossen hatte, für 6 Monate an einer Rotation in das AugenCentrum Erkelenz im Rahmen meiner Facharztweiterbildung teilzunehmen.

Diese optionale 6-monatige Praxisrotation integrierte die Uniklinik Köln 2017 in die 5-jährige Facharztausbildung zum $\mathrm{Au}$ genarzt. Der Arbeitsvertrag der Uniklinik läuft während der Rotation weiter, man wird beurlaubt und es erfolgt eine Anstellung im Augencentrum für die Rotation. Ich war die zweite Person, die an dem Kooperationsprojekt teilnahm. In einem für mich neuen Arbeitsumfeld wartete zwischen Mai und November 2018 eine spannende und v.a. lehrreiche Zeit auf mich.

\section{Arbeitsalltag in der Praxis - von} Anfang an voll integriert

Der Einstieg in den Arbeitsalltag war sehr rasant. Eine Kollegin war krank, sodass ich direkt lernte, was eine voll einbestellte Sprechstunde für einen Arzt in der Praxis bedeutet. Die Vorstellung eines viel entspannteren Arbeitsalltages in der Praxis musste ich also zügig revidieren. Die Menge an einem Tag zu behandelnder Patienten war für mich bis dahin unvorstellbar, alle 10 min war ein Patient terminiert. Am Anfang stellte dies für mich eine große Herausforderung dar. Dazu kamen noch das schlechte Gewissen, wenn die mir zugeteilte medizinische Fachangestellte (MFA) auf wertvolle Minuten der Mittagspause verzichten musste, und die Unzu- friedenheit der Patienten, die keine Wartezeiten in Kauf nehmen wollten. So war es unumgänglich, sich auf die wesentlichen Untersuchungen und ein möglichst zielgerichtetes Patientengespräch zu konzentrieren. Diese Effizienz war überhaupt nur möglich durch die gute Planung und die Unterstützung durch die mir zugeteilte MFA, die mitschrieb, Rezepte ausstellte, Briefe anfertigte und mich sogar mit Cappuccino verwöhnte. Was für ein Leben!

\section{Entscheidungsfindung in Klink und Praxis}

Obwohl ich bereits im dritten Weiterbildungsjahr war, merkte ich schnell, dass ich Sachverhalte viel intensiver hinterfragte, da ich nun eigenverantwortlich $\mathrm{Pa}$ tienten und Patientinnen behandelte und kein Oberarzt, wie aus der Klinik gewohnt, mir die endgültige Entscheidung abnahm. Als Entscheidungsgrundlage waren zwar diverse diagnostische Optionen in der Praxis vorhanden, ob man jedoch wirklich die betroffenen Patienten und Patientinnen von einer IGEL-Leistung überzeugen möchte, sollte vorher gut überlegt werden. Hier musste ich stets abwägen, ob die anfallenden Kosten den diagnostischen Nutzen aufwiegen. Da man in der Uniklinik das komplette diagnostische Programm anwenden kann, war dies eine Limitation, die ich nicht gewohnt war und die mich vor neue Herausforderungen stellte. Bei fachlichen Fragen standen mir jedoch auch immer kompetente und nette Kollegen und Kolleginnen zu Verfügung, die mich geduldig unterstützten. Allerdings empfehle ich trotzdem, erst ab dem dritten Weiterbildungsjahr an der Praxisrotation teilzunehmen, wenn man bereits klinische Erfahrung gesammelt hat.

\section{Neue operative Einblicke}

Ein Highlight meiner Praxisrotation war der Mittwoch. Denn mittwochs durfte ich unter Anleitung kleine operative Eingriffe durchführen und intravitreale Injektionen verabreichen. Dabei gab es auch Momente, in denen ich erfuhr, wie sich schwierige Situationen während eines Eingriffs anfühlen, und hatte die Gelegenheit, Strategien zu entwickeln, diese erfolgreich zu meistern. Besonders spannend war für mich, das Resultat des Eingriffs bei postoperativen Kontrollen zu sehen.

\section{Rundum lohnende Erfahrung} Abschließend kann ich auf die Frage „Wieso denn jetzt in die Praxis, du bist doch an der Uniklinik?" antworten, weil es mich unglaublich weitergebracht hat - und dazu einfach eine schöne Zeit war.

Vielen Dank an das ganze Team aus Erkelenz für die schöne gemeinsame Zeit! 